\title{
Estudio de la carga interna y externa a través de diferentes instrumentos. Un estudio de casos en fútbol formativo
}

\section{Study of internal and external load by different instruments. a case study in grassroots}

\author{
Gómez-Carmona, C.D. ${ }^{1}$; Gamonales, J.M. ${ }^{1}$; Feu, S. ${ }^{1}$; Ibáñez, S.J. ${ }^{1}$ \\ ${ }^{1}$ Grupo de Optimización del Entrenamiento y el Rendimiento Deportivo (GOERD). \\ Universidad de Extremadura, España.
}

Autor de correspondencia: Carlos David Gómez Carmona. Contacto: cdgomezcarmona@unex.es

\author{
Cronograma editorial: Artículo recibido: 04/07/2019 Aceptado: 14/08/2019 Publicado: 01/09/2019 \\ DOI: https://doi.org/10.17979/sportis.2019.5.3.5464
}

Financiación: Este trabajo ha sido parcialmente subvencionado por la Ayuda a los Grupos de Investigación (GR18170) de la Junta de Extremadura (Consejería de Empleo e Infraestructuras); con la aportación de la Unión Europea a través de los Fondos Europeos de Desarrollo Regional (FEDER).

Conflicto de intereses: Trabajo desarrollado dentro del Grupo de Optimización del Entrenamiento y Rendimiento Deportivo (GOERD) de la Facultad de Ciencias del Deporte de la Universidad de Extremadura. Todos los autores hemos contribuido en la realización del manuscrito y certificamos que no ha sido publicado ni está en vías de consideración para su publicación en otra revista. Aceptamos las normas de publicación y transferimos los derechos del presente trabajo a Sportis: Revista Técnico-Científica del Deporte Escolar, Educación Física y Psicomotricidad con ISBN 2386-8333, firmado por todos los autores.

Para citar este artículo utilice la siguiente referencia: Gómez-Carmona, C.D.; Gamonales, J.M.; Feu, S.; Ibáñez, S.J. (2019). Estudio de la carga interna y externa a través de diferentes instrumentos. Un estudio de casos en fútbol formativo. Sportis Sci J, 5 (3), 444-468. 


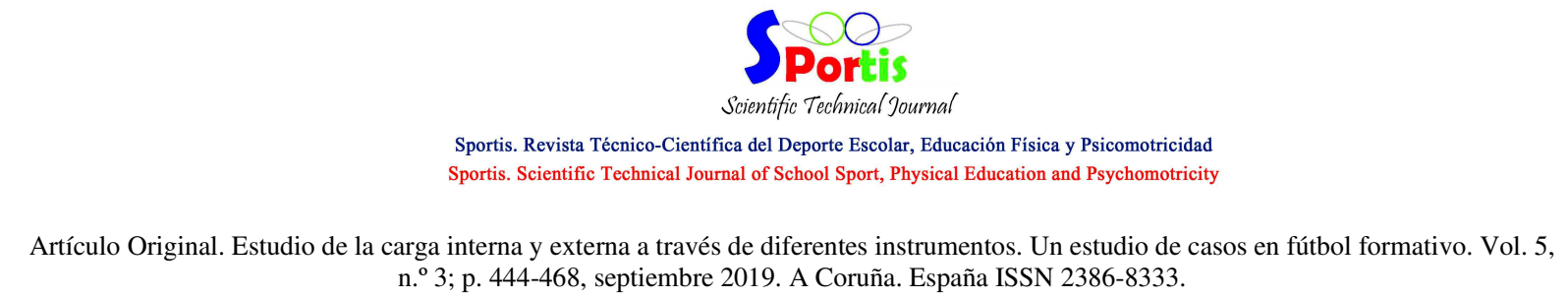

\section{Resumen}

Los dispositivos tecnológicos utilizados actualmente para registrar carga externa e interna no son accesibles al fútbol formativo por su alto coste económico. Por ello, herramientas para cuantificar la carga y categorizar las tareas son muy útiles para el proceso de entrenamiento. Por tanto, los objetivos del presente trabajo son: (1) identificar las diferencias en la carga objetiva en función de las variables de carga externa subjetiva y (2) analizar la relación entre ambos métodos de cuantificación. 157 tareas realizadas por un equipo de 20 jugadores juveniles fueron analizadas. La recogida de datos se realizó mediante: (a) carga objetiva, dispositivos inerciales WIMU $^{\mathrm{TM}}$ y (b) carga subjetiva, Sistema Integral para el Análisis de las Tareas de Entrenamiento (SIATE). El análisis estadístico estuvo compuesto por ANOVA de un factor con el post-hoc Bonferroni y la prueba de correlación de Pearson. Los principales resultados indican que existe influencia de las variables de carga subjetiva en las variables de carga objetiva $(p<.05)$, excepto en el porcentaje de ejecutantes simultáneos ( $p>.05)$, encontrando una alta correlación entre ellas $(r>.84)$. Las características de las tareas modifican de forma directa las demandas de carga, siendo herramientas subjetivas como SIATE útiles para su registro ante la ausencia de material tecnológico.

\section{Palabras clave}

SIATE; dispositivos inerciales; demandas cinemáticas; carga interna.

\section{Abstract}

The technological devices utilized currently for the external and internal load register are not accessible in grassroots due to their high costs. Thus, tools for load quantification and tasks categorization are very useful to improve the training process. Therefore, the aims of the present study are to: (1) identify the differences between objective load of training tasks in relation to subjective external load variables and (2) analyse the relationship between both load quantification methods. 157 tasks performed by an 18-players youth soccer team were analysed. The load register was realised by: (a) objective load, WIMU ${ }^{\mathrm{TM}}$ inertial devices and

Para citar este artículo utilice la siguiente referencia: Gómez-Carmona, C.D.; Gamonales, J.M.; Feu, S.; Ibáñez, S.J. (2019). Estudio de la carga interna y externa a través de diferentes instrumentos. Un estudio de casos en fútbol formativo. Sportis Sci J, 5 (3), 444-468. 
Artículo Original. Estudio de la carga interna y externa a través de diferentes instrumentos. Un estudio de casos en fútbol formativo. Vol. 5 , n. ${ }^{\circ}$; p. 444-468, septiembre 2019. A Coruña. España ISSN 2386-8333.

(b) subjective load, Integral System for Training Tasks Analysis (SIATE). The statistical analysis was composed by one-way ANOVA with Bonferroni post-hoc and Pearson correlation test. The main results indicate that exist an influence of subjective load variables in objective load variables $(p<.05)$, except in simultaneous player percentage $(p>.05)$, finding a high correlation between loads $(r>.84)$. The task characteristics directly modify the load demands, being subjective tools as SIATE useful for their register when technological devices are not available.
\end{abstract}

\title{
Keywords
}

SIATE; inertial devices; kinematical demands; internal load.

\section{Introducción}

La planificación de tareas de entrenamiento en los deportes de invasión ha evolucionado de manera significativa en los últimos años (Ibáñez, Feu, y Cañadas, 2016), desde una metodología tradicional hacia metodologías más activas y participativas (GonzálezEspinosa, Ibáñez, Feu, y Galatti, 2017), donde los jugadores son conscientes de su propio proceso de enseñanza-aprendizaje (Cañadas, Rodríguez, Feu, Parejo, y García, 2013). El análisis de las tareas de entrenamiento en el fútbol-base permitirá estudiar el posicionamiento metodológico, así como generar nuevas teorías de entrenamiento basadas en la práctica (Gamonales et al., 2019).

La obtención de datos cuantitativos permite mejorar el proceso de entrenamiento en todos sus ámbitos (Akenhead y Nassis, 2016). Por ello, el análisis de la carga externa (Buchheit, Lacome, Cholley, y Simpson, 2018), carga interna (fisiológica) (Achten y Jeukendrup, 2003), la disposición táctica (Reche-Soto, Cardona-Nieto, Diaz-Suarez, GómezCarmona, y Pino-Ortega, 2019) y los factores contextuales (edad relativa, nivel competitivo, periodo de juego, calendario competitivo, puesto específico, entre otros) (Arruda et al., 2015; Pino-Ortega et al., 2019) es importante para cuantificar las demandas específicas durante la

Para citar este artículo utilice la siguiente referencia: Gómez-Carmona, C.D.; Gamonales, J.M.; Feu, S.; Ibáñez, S.J. (2019). Estudio de la carga interna y externa a través de diferentes instrumentos. Un estudio de casos en fútbol formativo. Sportis Sci J, 5 (3), 444-468. 
Artículo Original. Estudio de la carga interna y externa a través de diferentes instrumentos. Un estudio de casos en fútbol formativo. Vol. 5 , n. ${ }^{\circ}$; p. 444-468, septiembre 2019. A Coruña. España ISSN 2386-8333.

competición y diseñar tareas que respondan a estas exigencias durante las sesiones de entrenamiento (Gómez-Carmona, Gamonales, Pino-Ortega, y Ibáñez, 2018).
\end{abstract}

En este sentido, dispositivos electrónicos para el análisis del rendimiento (EPTS) han sido desarrollados para su registro. Estos dispositivos están compuestos por diferentes sensores (acelerómetros, giróscopos, magnetómetros, GPS, LPM, entre otros) (Cummins, Orr, O’Connor, y West, 2013). En los últimos años, gracias al creciente interés investigador, numerosas investigaciones han mostrado su utilidad para identificar las exigencias en variables de tiempo-movimiento (Casamichana y Castellano, 2015; Gómez-Carmona, Gamonales, Pino-Ortega, y Ibáñez, 2018), la carga neuromuscular (Casamichana, Castellano, Calleja-Gonzalez, San Román, y Castagna, 2013; Reche-Soto et al., 2018), disposición táctica (Bastida-Castillo, Gómez-Carmona, de la Cruz Sánchez, y Pino-Ortega, 2019) y la carga interna mediante frecuencia cardíaca (Castellano, Puente, Echeazarra, Usabiaga, y Casamichana, 2016; Vilar, Duarte, Silva, Chow, y Davids, 2014).

Debido al alto coste de estos dispositivos, diferentes herramientas sin coste han sido desarrolladas, siendo la percepción subjetiva del esfuerzo (RPE) la que tiene un uso más extendido (Abbott, Brickley, y Smeeton, 2018; Casamichana et al., 2013). Posteriormente, herramientas para el análisis de las características de las tareas han sido desarrolladas, entre las que se encuentra el instrumento Sistema Integral para el Análisis de las Tareas de Entrenamiento (SIATE) (Ibáñez, Feu y Cañadas, 2016). Esta herramienta permite conocer en fútbol formativo las situaciones de juego empleadas por los entrenadores (Gamonales, Gómez-Carmona, León, Muñoz-Jiménez, y Ibáñez, 2020), los contenidos y medios de entrenamiento (Gamonales, Gómez-Carmona, Córdoba-Caro, y Ibáñez, 2019), las fases de juego (Gómez-Carmona, García-Rubio, Muñoz-Jiménez, y Gamonales, 2018), la relación entre las variables pedagógicas del entrenamiento (Gamonales et al., 2020) o la carga durante una tarea (Gracia, Garcia, Cañadas, y Ibáñez, 2014) a lo largo de la temporada.

Actualmente existe un déficit en la investigación de la relación existente entre herramientas de cuantificación de la carga subjetiva, a excepción de la percepción subjetiva Para citar este artículo utilice la siguiente referencia: Gómez-Carmona, C.D.; Gamonales, J.M.; Feu, S.; Ibáñez, S.J. (2019). Estudio de la carga interna y externa a través de diferentes instrumentos. Un estudio de casos en fútbol formativo. Sportis Sci J, 5 (3), 444-468. 
Artículo Original. Estudio de la carga interna y externa a través de diferentes instrumentos. Un estudio de casos en fútbol formativo. Vol. 5 , n. ${ }^{\circ}$; p. 444-468, septiembre 2019. A Coruña. España ISSN 2386-8333.

del esfuerzo, y la carga objetiva para su registro de en situaciones de entrenamiento y competición en deportes colectivos. A partir de lo expuesto, se hipotetiza que las variables de carga externa subjetiva condicionan la carga externa objetiva y que existirá una relación entre las mediciones de carga objetiva y subjetiva facilitadas por las herramientas empleadas en el estudio. Por tanto, los objetivos de la presente investigación fueron: (1) identificar las diferencias de carga objetiva en función de las variables de carga externa subjetivas registradas mediante la herramienta SIATE, y (2) analizar la relación entre la carga externa subjetiva y la carga objetiva durante las tareas llevadas a cabo durante tres meses de entrenamiento en fútbol formativo, en la categoría juvenil.
\end{abstract}

\title{
Método
}

\section{Diseño}

El diseño del presente estudio se encuadra dentro de la investigación correlacional, en la que se estudia las relaciones entre variables con el objetivo de predecir una variable categórica y de los estudios transversales con grupos naturales con el objetivo de caracterizar la carga interna y externa objetiva de las tareas de entrenamiento y comparar sus demandas en función de la carga externa subjetiva (Ato, López-García, y Benavente, 2013). No existe manipulación de variables ni administración de tratamiento experimental, teniendo la investigación un tratamiento ecológico.

\section{Muestra y Participantes}

Se analizaron 23 sesiones de entrenamiento correspondientes a tres meses de competición oficial durante la temporada 2017-2018, comprendiéndose entre los meses de enero $(n=9)$, febrero $(n=8)$ y marzo $(n=6)$. El equipo analizado de categoría sub-19 participaba en el campeonato regional de Liga de Primera División Regional Juvenil, organizado por la Federación de Fútbol Autonómica. El registro dio lugar a un total de 157 tareas las cuales fueron diseñadas, elaboradas y desarrolladas por un único entrenador, el cual

Para citar este artículo utilice la siguiente referencia: Gómez-Carmona, C.D.; Gamonales, J.M.; Feu, S.; Ibáñez, S.J. (2019). Estudio de la carga interna y externa a través de diferentes instrumentos. Un estudio de casos en fútbol formativo. Sportis Sci J, 5 (3), 444-468. 
Artículo Original. Estudio de la carga interna y externa a través de diferentes instrumentos. Un estudio de casos en fútbol formativo. Vol. 5 , n. ${ }^{\circ}$; p. 444-468, septiembre 2019. A Coruña. España ISSN 2386-8333.

presentaba una experiencia como jugador mayor de 25 años y como entrenador de 9 años. Además, posee como formación académica el grado de Doctor en Ciencias del Deporte y a nivel deportivo el título de Técnico Deportivo en Fútbol (Nivel II).
\end{abstract}

En el presente estudio participaron 20 jugadores de fútbol varones de categoría juvenil (edad: 17.32 \pm 0.87 años; masa corporal: $67.27 \pm 5.78 \mathrm{~kg}$; altura: $1.72 \pm 0.08$ metros; IMC: $21.83 \pm 1.89 \mathrm{~kg} / \mathrm{m}^{2}$ ). Los participantes presentaron los siguientes criterios de inclusión: (1) no padecer limitaciones o lesiones musculoesqueléticas que impidieran la práctica deportiva, y (2) participación en el total de la sesión de entrenamiento. Los porteros fueron excluidos del estudio debido a que la carga física difiere con respecto al resto de jugadores de campo (White et al., 2018). Por tanto, 18 jugadores fueron analizados.

Tanto el cuerpo técnico como los jugadores fueron informados previamente de los detalles de la investigación y de sus posibles riesgos y beneficios, por lo que para ello se les suministró un consentimiento informado. En los jugadores menores de edad, el consentimiento fue suscrito por sus tutores legales. El estudio se desarrolló en base al Código Ético de la Asociación Médica Mundial y las disposiciones éticas de la Declaración de Helsinki (2013), siendo aprobado por el Comité de Bioética de la Universidad de Extremadura ( $n^{\circ}$ registro 67/2017). El club autorizó todos los protocolos de actuación.

\title{
Instrumentos y variables analizadas
}

\section{Carga externa y carga interna objetiva}

Las variables de carga externa registradas fueron distancia total (Dellaserra, Gao, y Ransdell, 2014), distancia a alta intensidad (>16 km/h) (Bradley et al., 2009), aceleraciones (Arruda et al., 2015) y Player Load (Reche-Soto et al., 2018), siendo analizadas de forma total y relativas al tiempo de participación. Además, se registró para el análisis de la carga interna la frecuencia cardíaca (Achten y Jeukendrup, 2003).

Para citar este artículo utilice la siguiente referencia: Gómez-Carmona, C.D.; Gamonales, J.M.; Feu, S.; Ibáñez, S.J. (2019). Estudio de la carga interna y externa a través de diferentes instrumentos. Un estudio de casos en fútbol formativo. Sportis Sci J, 5 (3), 444-468. 
Artículo Original. Estudio de la carga interna y externa a través de diferentes instrumentos. Un estudio de casos en fútbol formativo. Vol. 5 , n. ${ }^{\circ}$; p. 444-468, septiembre 2019. A Coruña. España ISSN 2386-8333.

Tabla 1. Descripción de las variables objetivas de carga interna y externa.

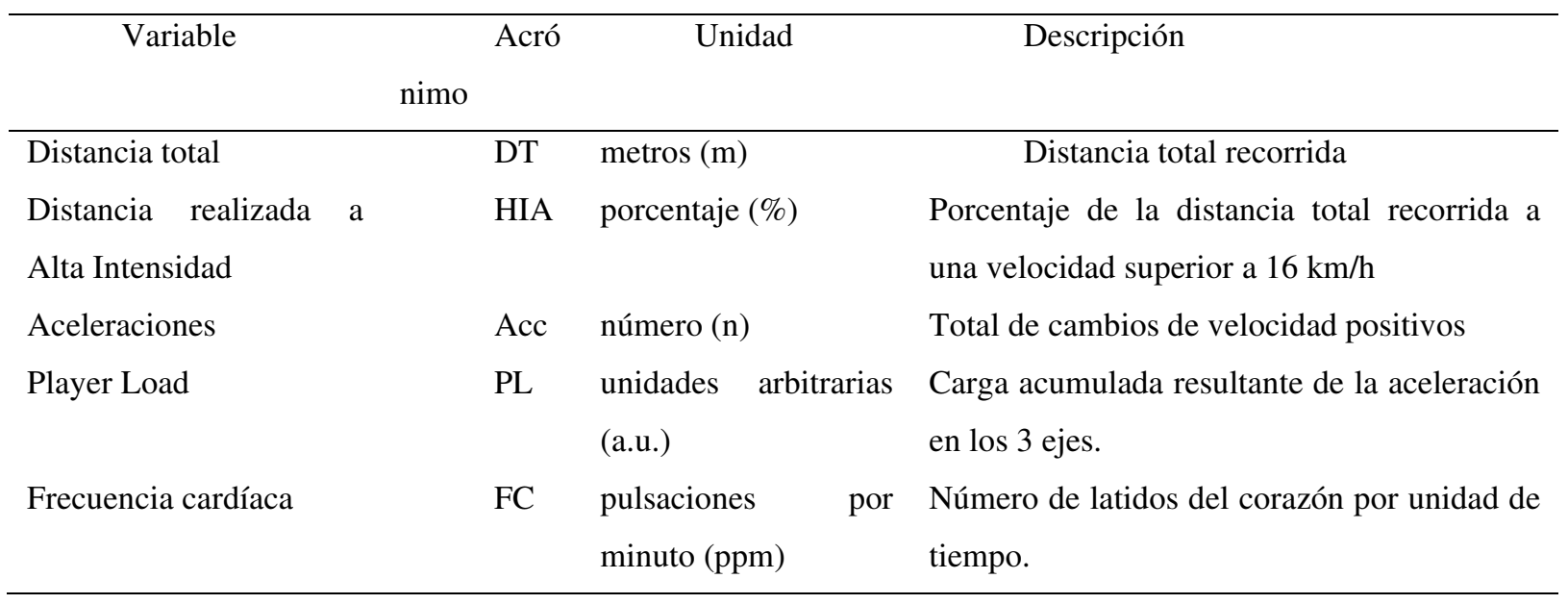

Para su registro se utilizaron dispositivos inerciales WIMU ${ }^{\mathrm{TM}}$ (RealTrack Systems, Almería, España), compuestos por diferentes sensores (cuatro acelerómetros triaxiales; tres giróscopos, un magnetómetro, sensores UWB y GPS). La precisión del dispositivo ha sido evaluada previamente para localización en condiciones de interior y exterior (BastidaCastillo, Gómez-Carmona, De la Cruz Sánchez, y Pino-Ortega, 2018; Bastida-Castillo, Gómez-Carmona, Hernandez, y Pino-Ortega, 2018) y para la carga acelerométrica (GomezCarmona, Bastida-Castillo, González-Custodio, Olcina, y Pino-Ortega, 2019; GómezCarmona, Bastida-Castillo, García-Rubio, Ibáñez, y Pino-Ortega, 2019).

Las demandas de carga externa fueron registradas mediante: (a) GPS para los datos de localización en exterior con una frecuencia de muestreo de $5 \mathrm{~Hz}$, y (b) acelerómetro para la carga del movimiento del participante en función de la gravedad mediante una frecuencia de muestreo de $100 \mathrm{~Hz}$. La frecuencia cardíaca (FC) fue utilizada para el registro de la carga interna mediante una banda de FC GARMIN ${ }^{\mathrm{TM}}$ (Garmin Ltd., Olathe, Kansas, EEUU), que enviaba los datos al dispositivo inercial mediante tecnología Ant+ (Molina-Carmona, GómezCarmona, Bastida Castillo, y Pino-Ortega, 2018). Durante la presente investigación, la información fue registrada en la memoria interna de $8 \mathrm{~GB}$ que posee cada dispositivo.

Para citar este artículo utilice la siguiente referencia: Gómez-Carmona, C.D.; Gamonales, J.M.; Feu, S.; Ibáñez, S.J. (2019). Estudio de la carga interna y externa a través de diferentes instrumentos. Un estudio de casos en fútbol formativo. Sportis Sci J, 5 (3), 444-468. 
Artículo Original. Estudio de la carga interna y externa a través de diferentes instrumentos. Un estudio de casos en fútbol formativo. Vol. 5 , n. ${ }^{\circ}$; p. 444-468, septiembre 2019. A Coruña. España ISSN 2386-8333.

\section{Carga externa subjetiva}

Fue registrada mediante el Sistema Integral para el Análisis de las Tareas de Entrenamiento (SIATE), el cual es un sistema metodológico para registrar y analizar los diferentes factores que inciden en el entrenamiento en deportes de invasión. Es una herramienta modulable ya que se puede definir la información que los entrenadores registran de cada tarea. Por tanto, cuanto mayor información se consiga obtener de cada tarea más profundo podrá ser el análisis y la evaluación de la misma (Ibáñez et al., 2016).

Para el cálculo de la carga externa subjetiva, en primer lugar, se registran seis variables primarias definidas por Ibáñez et al. (2016): Grado de Oposición, Densidad de la Tarea, Porcentaje de Ejecutantes Simultáneos, Carga Competitiva, Espacio de Juego e Implicación Cognitiva. Todas las variables se categorizan en cinco niveles, ordenados de menor a mayor carga, desde 1 (carga mínima) a 5 (carga máxima), mostrándose en la tabla 2.

Tabla 2. Variables de carga externa registradas a través de la herramienta SIATE.

\begin{tabular}{lll}
\hline Variable & \multicolumn{1}{c}{ Definición } & Categoría \\
\hline Grado de & Carga que supone la tarea & (1) Sin oposición \\
oposición & para los deportistas en & (2) Superioridad $>3$ deportistas \\
& función del número de & (3) Superioridad 2 deportistas; \\
& oponentes. & (4) Superioridad 1 deportista \\
& (5) Igualdad numérica
\end{tabular}

Para citar este artículo utilice la siguiente referencia: Gómez-Carmona, C.D.; Gamonales, J.M.; Feu, S.; Ibáñez, S.J. (2019). Estudio de la carga interna y externa a través de diferentes instrumentos. Un estudio de casos en fútbol formativo. Sportis Sci J, 5 (3), 444-468. 
Artículo Original. Estudio de la carga interna y externa a través de diferentes instrumentos. Un estudio de casos en fútbol formativo. Vol. 5 n. ${ }^{\circ} 3$; p. 444-468, septiembre 2019. A Coruña. España ISSN 2386-8333.

\begin{tabular}{lllll}
\hline ejecutantes & deportistas & durante & la & (3) $41-60 \%$ \\
simultáneos & tarea & & (4) $61-80 \%$ \\
& & & (5) Superior al $81 \%$. \\
\hline
\end{tabular}

\begin{tabular}{|c|c|c|}
\hline Carga & Carga psicológica que & (1) Actividad en la que no se compite \\
\hline
\end{tabular}
debido a la presión por el técnicos

resultado (3) Actividades con oposición sin contabilizar

(4) Actividades de oposición reducida contabilizando el resultado

(5) Partidos con resultado y equipo completo

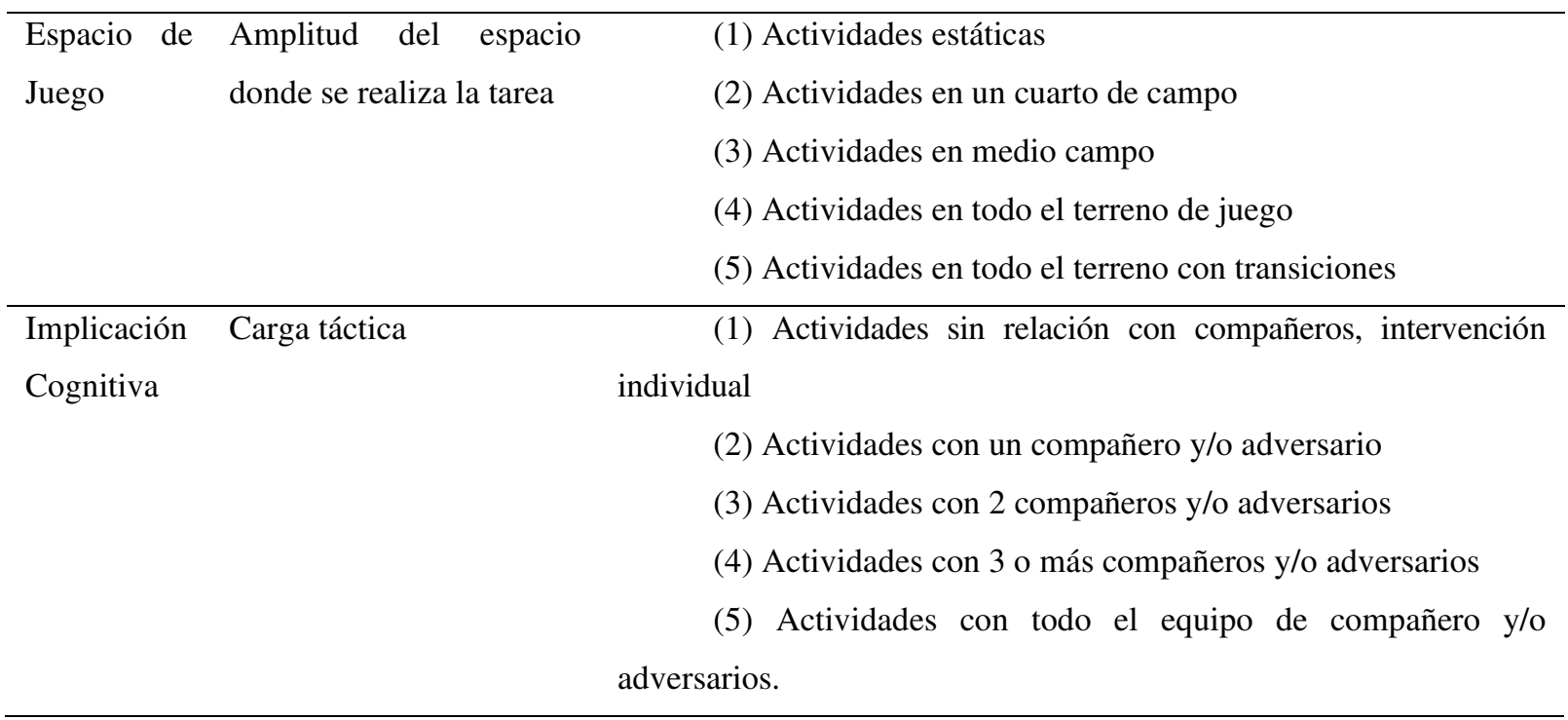

A partir de estas categorías se calcula:

- Carga de la Tarea (CT): Esta variable se calcula a partir del sumatorio del valor asignado a cada una de las seis variables anteriores (1 a 5 puntos). Su valor es una escala, que tiene un rango desde 6 a 30 Unidades de Carga, siendo el valor 6 como carga muy baja y el valor 30 como carga muy alta.

- Carga de la tarea por tiempo (CTT): Identifica la carga de la tarea en relación con el tiempo de ejecución, obteniendo un valor ajustado de cada una de las tareas de entrenamiento en relación con el tiempo real de práctica. Es calculado multiplicando la CT por el tiempo útil que han estado

Para citar este artículo utilice la siguiente referencia: Gómez-Carmona, C.D.; Gamonales, J.M.; Feu, S.; Ibáñez, S.J. (2019). Estudio de la carga interna y externa a través de diferentes instrumentos. Un estudio de casos en fútbol formativo. Sportis Sci J, 5 (3), 444-468. 
Artículo Original. Estudio de la carga interna y externa a través de diferentes instrumentos. Un estudio de casos en fútbol formativo. Vol. 5 , n. ${ }^{\circ}$; p. 444-468, septiembre 2019. A Coruña. España ISSN 2386-8333.

practicando los deportistas medido en segundos. Este valor es el que se utilizará en el análisis de datos.
\end{abstract}

\title{
Procedimiento
}

El equipo fue registrado dos días por semana, martes y jueves, durante las sesiones de entrenamiento. Las sesiones tenían una duración de 1 hora y 30 minutos. Para el registro de la carga externa e interna objetiva, los jugadores eran citados 15 minutos antes del comienzo de cada sesión de entrenamiento para ubicar los dispositivos inerciales y la banda de frecuencia cardíaca. Previamente a su colocación, los dispositivos fueron calibrados y sincronizados siguiendo las recomendaciones del fabricante, el cual se realiza en la configuración interna del arranque. Para el autocalibrado se tuvo en cuenta 3 aspectos: (i) dejar el dispositivo inmóvil durante 30 segundos, (ii) situarlo en una zona plana y (iii) sin dispositivos magnéticos alrededor. Posteriormente, los dispositivos fueron colocados en cada jugador mediante un arnés ajustado anatómicamente. Durante la sesión, la selección de la duración de las actividades fue realizada en tiempo real mediante el software SVIVO ${ }^{\mathrm{TM}}$ (RealTrack Systems, Almería, España). Los datos obtenidos por los dispositivos fueron sincronizados y extraídos para su posterior análisis mediante el software $\mathrm{S} \mathrm{PRO}^{\mathrm{TM}}$ (RealTrack Systems, Almería, España).

Para el registro de la carga externa subjetiva, previamente al inicio del registro, se formaron a dos observadores externos, para categorizar las tareas de entrenamiento mediante la herramienta SIATE. El proceso aplicado fue similar a los existentes en la literatura científica en otros contextos deportivos como en Fútbol a 5 para personas ciegas (Gamonales, Muñoz-Jiménez, León, y Ibáñez, 2017) o fútbol (Gamonales et al., 2019).

\section{Análisis estadístico}

En primer lugar, se realizó un análisis descriptivo mostrando los datos como media y desviación estándar (media \pm DS) para describir las exigencias de carga interna y externa

Para citar este artículo utilice la siguiente referencia: Gómez-Carmona, C.D.; Gamonales, J.M.; Feu, S.; Ibáñez, S.J. (2019). Estudio de la carga interna y externa a través de diferentes instrumentos. Un estudio de casos en fútbol formativo. Sportis Sci J, 5 (3), 444-468. 


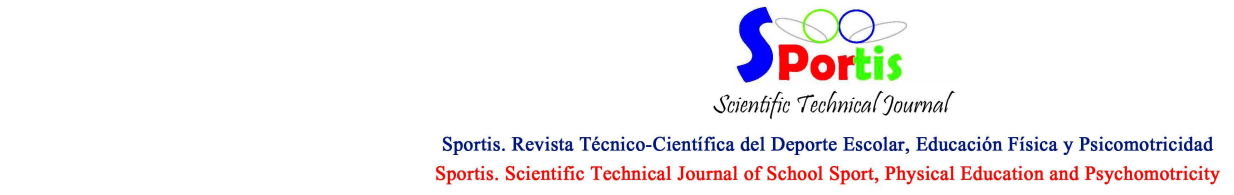

Artículo Original. Estudio de la carga interna y externa a través de diferentes instrumentos. Un estudio de casos en fútbol formativo. Vol. 5 , n. ${ }^{\circ}$; p. 444-468, septiembre 2019. A Coruña. España ISSN 2386-8333.

objetiva (distancia, distancia a alta intensidad, aceleraciones, player load y frecuencia cardíaca) en las diferentes tareas analizadas en función de las variables de carga externa subjetiva (nivel de oposición, densidad, ejecutantes simultáneos, carga competitiva, espacio de juego e implicación cognitiva). Para determinar la normalidad de las variables, se realizó la prueba Kolmogorov-Smirnov y para analizar la homocedasticidad la prueba de Levene, reportando ambas una distribución normal (Field, 2013).

Para analizar las diferencias entre las variables de carga subjetiva y objetiva se utilizó la prueba ANOVA de un factor, realizándose la comparación post-hoc mediante Bonferroni. El nivel de significación se estableció con el valor de $p<0.05$. Para calcular la magnitud de las diferencias se utilizó el cálculo estadístico omega parcial al cuadrado $\left(\omega_{p}{ }^{2}\right)$ clasificado como: $>0.01$ pequeño; >0.06 moderado and >0.14 grande (Cohen, 1988). Finalmente, para el análisis relacional de las variables de carga externa subjetiva y objetiva se utilizó la prueba de correlación de Pearson, interpretada según Field (2013): insignificante $\left(r^{2}<0.1\right)$, pequeña $\left(0.1<\mathrm{r}^{2}<0.3\right)$, moderada $\left(0.3<\mathrm{r}^{2}<0.5\right)$, grande $\left(0.5<\mathrm{r}^{2}<0.7\right)$, muy grande $\left(0.7<\mathrm{r}^{2}<0.9\right)$, casi perfecta $\left(r^{2}>0.9\right)$ y perfecta $\left(r^{2}=1\right)$. El análisis estadístico fue realizado mediante el software SPSS versión 24.0. (SPSS Inc., Armonk NY, EE. UU.), y el diseño de las figuras mediante el software GraphPad Prism versión 7.0 (Graphpad Inc., La Jolla CA, EE. UU.).

\section{Resultados}

En la figura 1 se muestra el análisis descriptivo e inferencial de las variables de carga externa objetiva registradas a partir del dispositivo inercial WIMU ${ }^{\mathrm{TM}}$ con respecto a las variables de carga externa subjetiva registradas mediante la herramienta SIATE. En la variable distancia total recorrida se encuentran diferencias significativas en función de las 6 variables de carga externa subjetiva con un amplio rango $(p<.05 ; F=2.25-20.25)$. El tamaño del efecto fue grande en la variable grado de oposición $\left(\omega_{p}^{2}=.33\right)$, densidad $\left(\omega_{p}^{2}=.36\right)$, carga competitiva $\left(\omega_{p}^{2}=.39\right)$, espacio de juego $\left(\omega_{p}^{2}=.35\right)$ e implicación cognitiva $\left(\omega_{p}^{2}=.33\right)$, y con un tamaño del efecto bajo en la variable número de participantes simultáneos $\left(\omega_{p}^{2}=.05\right)$.

Para citar este artículo utilice la siguiente referencia: Gómez-Carmona, C.D.; Gamonales, J.M.; Feu, S.; Ibáñez, S.J. (2019). Estudio de la carga interna y externa a través de diferentes instrumentos. Un estudio de casos en fútbol formativo. Sportis Sci J, 5 (3), 444-468. 
En la variable porcentaje de distancia recorrida a alta intensidad (velocidad $>16 \mathrm{~km} / \mathrm{h}$ ) se encuentran diferencias significativas en todas las variables analizadas $(p<.05 ; F=2.25-$ $20.25)$, excepto en la variable porcentaje de ejecutantes simultáneos $\left(p=.42 ; F=.98 ; \omega_{p}^{2<.01)}\right.$. El tamaño del efecto fue grande en carga competitiva $\left(\omega_{p}{ }^{2}=.17\right)$; moderado en espacio de juego $\left(\omega_{p}^{2}=.14\right)$, grado de oposición $\left(\omega_{p}^{2}=.09\right)$ e implicación cognitiva $\left(\omega_{p}^{2}=.08\right)$; y pequeño en densidad $\left(\omega_{p}^{2}=.06\right)$. En cuanto a las aceleraciones por minuto, se encuentran diferencias significativas con un amplio rango $(p<.05 ; F=2.25-20.25)$ en las variables carga competitiva $\left(\omega_{p}^{2}=.24 ;\right.$ grande $)$, densidad $\left(\omega_{p}^{2}=.10 ;\right.$ moderado $)$, espacio de juego $\left(\omega_{p}^{2}=.05 ;\right.$ pequeño $)$ e implicación cognitiva $\left(\omega_{p}^{2}=.05\right.$; pequeño). No se encuentran diferencias significativas en el grado de oposición $\left(p=.31 ; F=1.22 ; \omega_{p}^{2}<0.01\right)$ y en el porcentaje de ejecutantes simultáneos $\left(p=.12 ; F=1.82 ; \omega_{p}^{2}=.02\right)$.

En relación con la variable Player Load se encuentran diferencias significativas en todas las variables analizadas $(p<.05 ; F=6.61-12.73)$, excepto en la variable porcentaje de ejecutantes simultáneos $\left(p=.09 ; F=2.02 ; \omega_{p}^{2}=.03\right)$. Todos los tamaños del efecto encontrados son con una magnitud alta (grado de oposición, $\omega_{p}{ }^{2}=.15$; densidad, $\omega_{p}{ }^{2}=.28$; carga competitiva, $\omega_{p}{ }^{2}=.19$; espacio de juego, $\omega_{p}^{2}=.22$; implicación cognitiva, $\omega_{p}^{2}=.16$ ). Finalmente, respecto a la carga interna registrada mediante la frecuencia cardíaca, se encuentran diferencias significativas con un alto tamaño del efecto en todas las variables analizadas $\left(p<.01 ; F>7.04 ; \omega_{p}^{2}>.17\right)$, excepto en la variable porcentaje de ejecutantes simultáneos $\left(p=.71 ; F=.53 ; \omega_{p}^{2<.01) .}\right.$ 


\section{Sportis}

Scientific Technical Journal

Sportis. Revista Técnico-Científica del Deporte Escolar, Educación Física y Psicomotricidad Sportis. Scientific Technical Journal of School Sport, Physical Education and Psychomotricity

Artículo Original. Estudio de la carga interna y externa a través de diferentes instrumentos. Un estudio de casos en fútbol formativo. Vol. 5, n. ${ }^{\circ}$; p. 444-468, septiembre 2019. A Coruña. España ISSN 2386-8333.

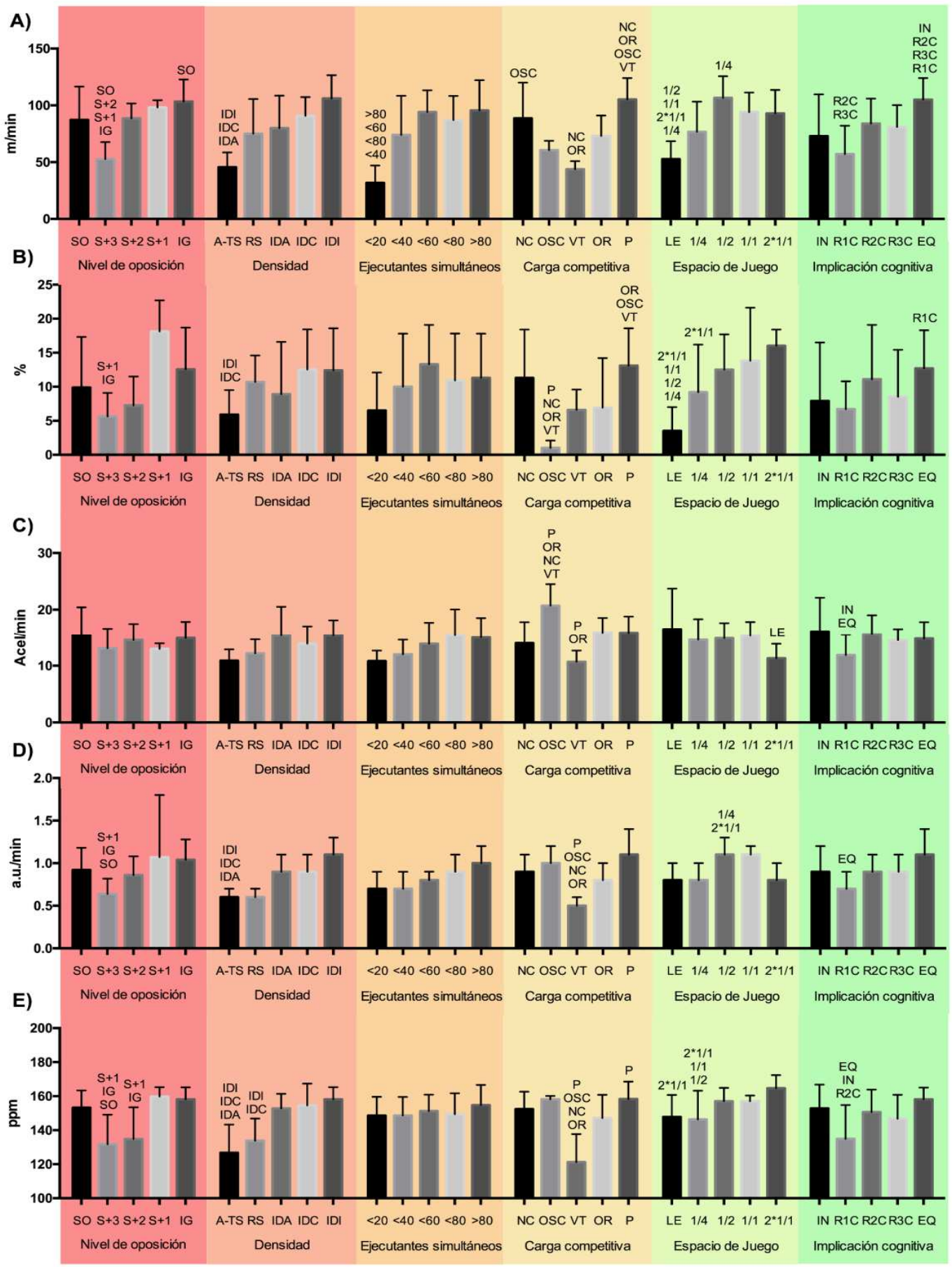

Figura 1. Representación gráfica del análisis descriptivo e inferencial de las variables de carga externa objetiva (A) distancia, (B) porcentaje de distancia a alta intensidad, (C) aceleraciones, (D) Player Load y (E) frecuencia cardíaca en función de las variables de carga externa subjetiva (1) Nivel de oposición (SO: sin oposición; S+3: superioridad +3; S+2: superioridad +2; S+1: superioridad +1; IG: igualdad), (2) Densidad Para citar este artículo utilice la siguiente referencia: Gómez-Carmona, C.D.; Gamonales, J.M.; Feu, S.; Ibáñez, S.J. (2019). Estudio de la carga interna y externa a través de diferentes instrumentos. Un estudio de casos en fútbol formativo. Sportis Sci J, 5 (3), 444-468. 
Artículo Original. Estudio de la carga interna y externa a través de diferentes instrumentos. Un estudio de casos en fútbol formativo. Vol. 5 , n. ${ }^{\circ}$; p. 444-468, septiembre 2019. A Coruña. España ISSN 2386-8333.

(A-TS: andando - trote suave; RS: ritmo suave; IDA: intensidad con descanso amplio ratio 1/2; IDC: intensidad con descanso completo, ratio 1/1; IDI: intensidad con descanso incompleto, ratio 2/1), (3) Ejecutantes simultáneos (<20: 0-20\%; <40: 20-40\%; <60: 40-60\%; <80: 60-80\%; >80: 80-100\%), (4) Carga competitiva (NC: No se compite; OSC: oposición sin contabilizar; VT: valoración técnica; OR: oposición reducida; P: partido), (5) Espacio de juego (LE: lugar específico; 1/4: cuarto de campo; 1/2: medio campo; 1/1: campo completo; $2 * 1 / 1$ : repetición de campo completo) y (6) Implicación cognitiva (IN: individual; R1C: relación con 1 compañero; $R 2 C$ : relación con 2 compañeros; $R 3 C$ : relación con 3 compañeros; EQ: relación con el equipo, 4 o más compañeros). El acrónimo encima de los gráficos de barras significa diferencias significativas con ese grupo $(p<.05)$.

En la figura 2, se muestran el análisis relacional entre las variables de carga externa subjetiva y objetiva registradas en la presente investigación. Se encuentran altos valores de correlación entre las variables analizadas $(r>.84 ; p<.05)$.

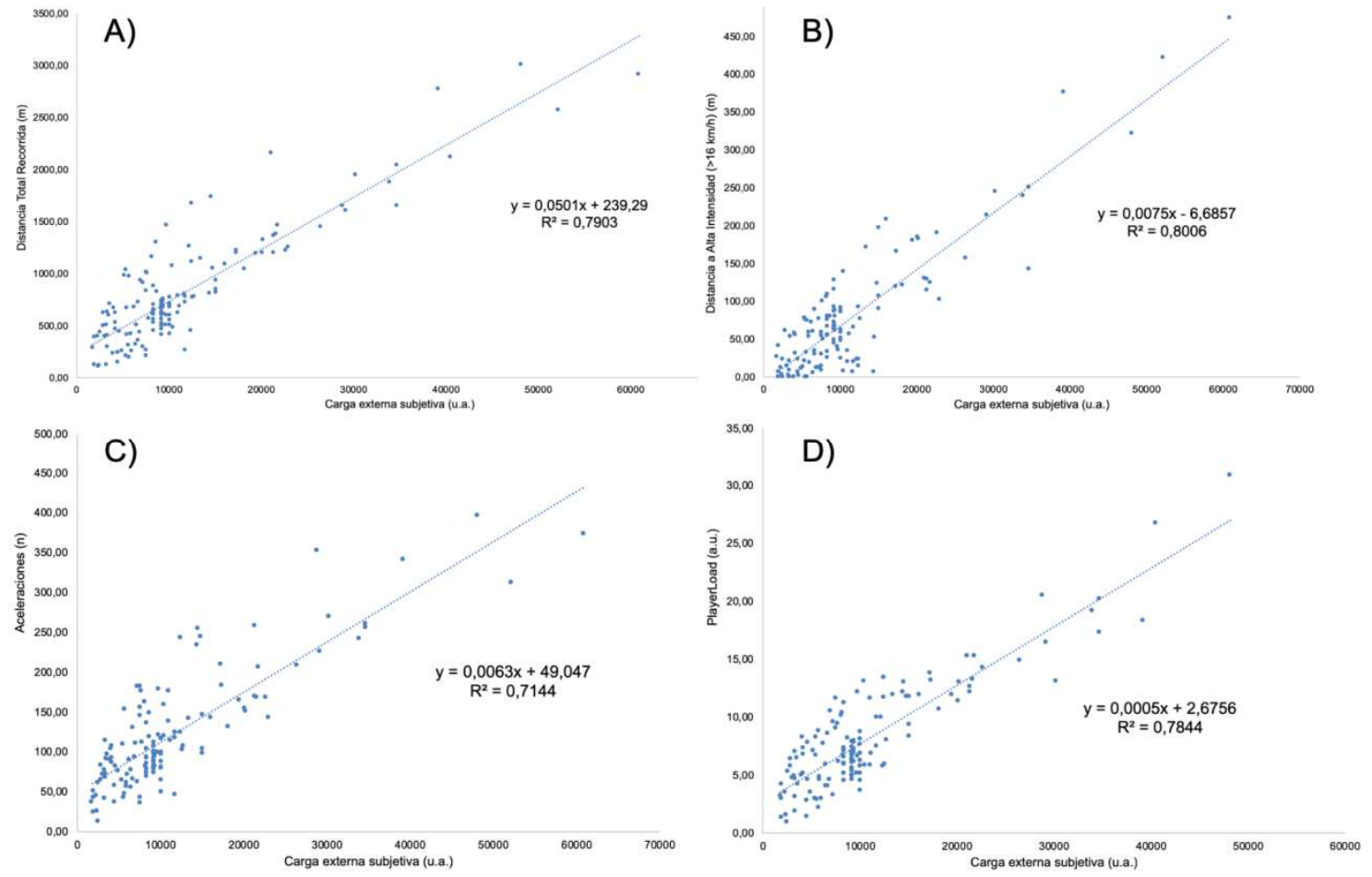

Figura 2. Gráfico de dispersión donde se muestra el análisis relacional entre la carga externa subjetiva y las variables de carga externa objetiva (A) Distancia total, (B) Distancia a alta intensidad (>16 km/h), (C) Aceleraciones y (D) Player Load.

Para citar este artículo utilice la siguiente referencia: Gómez-Carmona, C.D.; Gamonales, J.M.; Feu, S.; Ibáñez, S.J. (2019). Estudio de la carga interna y externa a través de diferentes instrumentos. Un estudio de casos en fútbol formativo. Sportis Sci J, 5 (3), 444-468. 


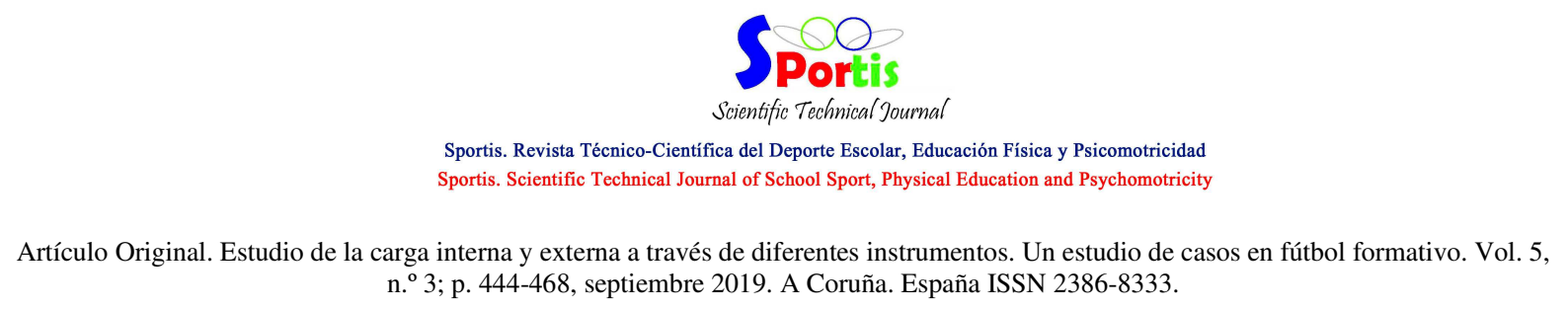

\section{Discusión}

Debido al alto coste de los dispositivos electrónicos para el análisis del rendimiento, herramientas objetivas para la medición de la carga se presentan como una alternativa, siendo necesario estudios previos que confirmen su precisión. Por tanto, los objetivos del presente estudio fueron analizar la influencia de las variables de carga externa subjetiva registradas mediante la herramienta SIATE con respecto a las variables de carga objetiva registradas por el dispositivo inercial WIMU ${ }^{\mathrm{TM}}$ e identificar la relación existente entre ambos indicadores de carga. Los resultados encontrados indican una fuerte influencia de las variables de carga externa subjetiva respecto a las variables de carga externa objetiva $(p<.05)$, excepto en la variable porcentaje de ejecutantes simultáneos (distancia total: $p=.05, \omega_{p}^{2}=.05$; distancia a alta intensidad: $p=.42, \omega_{p}^{2}=.01$; Player Load: $p=.09, \omega_{p}^{2}=.03$; aceleraciones: $p=.12, \omega_{p}^{2}=.02$; frecuencia cardíaca: $p=.71, \omega_{p}^{2}=.01$ ). Además, se encontró una relación muy fuerte entre las variables de carga objetiva y subjetiva $(r>.84)$.

\section{Nivel de Oposición}

El nivel de oposición provocó diferencias significativas en todas las variables analizadas con un tamaño del efecto grande, excepto en aceleraciones por minuto ( $p=.31$; $\left.F=1.22 ; \omega_{p}^{2}=.01\right)$. Se encuentran las mayores exigencias en las tareas de Superioridad $+1 \mathrm{e}$ Igualdad, mientras que las menores exigencias se encuentran en las tareas de Superioridad +3 . Torres-Ronda et al. (2015) han evaluado el efecto de jugar en superioridad, inferioridad baja e inferioridad alta durante juegos reducidos en fútbol, encontrando las menores exigencias en distancia recorrida, lactato y percepción subjetiva del esfuerzo en situaciones de alta inferioridad, al igual que en la presente investigación. Por ello, las situaciones de igualdad numérica o pequeña inferioridad (+1 jugador) generan las mayores exigencias. Las tareas con alta inferioridad (diferencia >3 jugadores) son situaciones idóneas para aumentar los estímulos del equipo en situación defensiva (jugadores suplentes o no convocados) y reducir los estímulos del equipo en situación ofensiva (jugadores titulares) en las sesiones postpartido.

Para citar este artículo utilice la siguiente referencia: Gómez-Carmona, C.D.; Gamonales, J.M.; Feu, S.; Ibáñez, S.J. (2019). Estudio de la carga interna y externa a través de diferentes instrumentos. Un estudio de casos en fútbol formativo. Sportis Sci J, 5 (3), 444-468. 
Artículo Original. Estudio de la carga interna y externa a través de diferentes instrumentos. Un estudio de casos en fútbol formativo. Vol. 5, n. ${ }^{\circ}$; p. 444-468, septiembre 2019. A Coruña. España ISSN 2386-8333.
\end{abstract}

\title{
Densidad y Ejecutantes Simultáneos
}

La intensidad en la realización de las tareas produjo diferencias significativas en todas las variables analizadas con un tamaño del efecto moderado en distancia a alta intensidad y aceleraciones por minuto y con un tamaño del efecto grande en distancia total, Player Load y frecuencia cardíaca. Se encuentran las mayores exigencias en las tareas con intensidad y recuperación completa e incompleta, mientras que las menores exigencias se encuentran en las tareas con la densidad andando/trote suave. Diferentes investigaciones recopiladas en una reciente revisión (Halouani, Chtourou, Gabbett, Chaouachi, y Chamari, 2014) han analizado el efecto de los períodos de recuperación en las actividades en fútbol, con especial hincapié en las tareas basadas en el juego, encontrando mayores exigencias en los juegos reducidos con duración continua respecto a los que seguían una estrategia intermitente. Además, en cuanto a

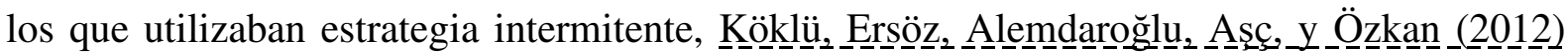
encontraron que una recuperación incompleta (ratio 2/1 o 4/1) provoca mayores exigencias de carga interna respecto a tareas con recuperación completa (ratio 1/1). Por tanto, la intensidad y el tiempo de recuperación entre series dentro de las tareas modifican las exigencias físicas y deben ser planificados de forma correcta para lograr las adaptaciones específicas en función del objetivo de la sesión. Para ello, una reducción del tiempo de recuperación y un aumento de la intensidad provoca mayores exigencias, encontrando los mayores efectos en las variables de volumen (distancia total, Player Load) con respecto a las variables de intensidad (aceleraciones y distancia a alta intensidad), debido a que no existe el tiempo suficiente para su recuperación.

El porcentaje de ejecutantes simultáneos únicamente modificó las exigencias en la variable distancia relativa $\left(p=.05 ; F=2.25 ; \omega_{p}{ }^{2}=.08\right)$, no encontrando diferencias en el resto de variables $\left(p>.09 ; F>.53 ; \omega_{p}^{2<.02)}\right.$. Un porcentaje menor del $20 \%$ reportó las menores demandas. Un aumento de este porcentaje consiguió unas demandas similares, logrando las mayores exigencias cuando el porcentaje de participación era máximo (80-100\%). Por tanto, un menor porcentaje de ejecutantes simultáneos, provoca, por ende, un mayor tiempo de

Para citar este artículo utilice la siguiente referencia: Gómez-Carmona, C.D.; Gamonales, J.M.; Feu, S.; Ibáñez, S.J. (2019). Estudio de la carga interna y externa a través de diferentes instrumentos. Un estudio de casos en fútbol formativo. Sportis Sci J, 5 (3), 444-468. 
Artículo Original. Estudio de la carga interna y externa a través de diferentes instrumentos. Un estudio de casos en fútbol formativo. Vol. 5 , n. ${ }^{\circ}$; p. 444-468, septiembre 2019. A Coruña. España ISSN 2386-8333.

recuperación, lo cual está en relación con una menor densidad y menores exigencias durante la realización de las tareas.
\end{abstract}

\title{
Carga competitiva y Espacio de Juego
}

En ambas variables, se encuentran diferencias significativas respecto a la carga externa objetiva (carga competitiva: $p<.01, F>6.93, \omega_{p}^{2}>.20$ efecto grande; espacio de juego: $p<.05 ; F>2.37 ; \omega_{p^{2}}>.08$ efecto moderado). En carga competitiva, los mayores valores se encuentran en las tareas donde no se compite o se disputa un partido, y los menores valores en las tareas de valoración técnica. Respecto al espacio de juego, los mayores valores se reportan cuando el espacio de juego es mayor de medio campo y los menores valores en ejecuciones en lugar específico (faltas, penaltis, etc.) en distancia, distancia a alta intensidad y frecuencia cardíaca. Este patrón es inverso con respecto a las exigencias en cambios de velocidad (aceleraciones y desaceleraciones), provocando un área más reducida mayores exigencias en estas variables.

Respecto a la carga competitiva, Gómez-Carmona, Gamonales, Pino-Ortega, y Ibáñez (2018) identificaron que el objetivo de la tarea influye de forma directa en las exigencias de los juegos reducidos, encontrando las mayores demandas cuando el objetivo era anotar gol en una portería con portero respecto a (1) posesión, (2) posesión y progresión, y (3) posesión, progresión y finalización en mini-metas. En este sentido, Halouani, Chtourou, Dellal, Chaouachi, y Chamari (2017) encontraron que los juegos de posesión y progresión presentaban mayores demandas que los juegos con finalización en minimetas, debido a tener que defender una mayor superficie y tener una mayor similitud con el juego real. Por tanto, una mayor carga competitiva y similitud con las condiciones de juego real determina unas mayores exigencias, excepto cuando se realizan tareas condicionales de forma individual. Para las sesiones de entrenamiento, es recomendable el uso de tareas con balón que simulen las exigencias competitivas respecto a las tareas condicionales aisladas sin balón porque ambas provocan las mismas adaptaciones físicas (Hill-Haas, Coutts, Rowsell, y Dawson,

Para citar este artículo utilice la siguiente referencia: Gómez-Carmona, C.D.; Gamonales, J.M.; Feu, S.; Ibáñez, S.J. (2019). Estudio de la carga interna y externa a través de diferentes instrumentos. Un estudio de casos en fútbol formativo. Sportis Sci J, 5 (3), 444-468. 
Artículo Original. Estudio de la carga interna y externa a través de diferentes instrumentos. Un estudio de casos en fútbol formativo. Vol. 5 , n. ${ }^{\circ}$; p. 444-468, septiembre 2019. A Coruña. España ISSN 2386-8333.
\end{abstract}

2009), eliminando las tareas condicionales el trabajo de los requerimientos técnico-tácticos inherentes en el rendimiento en los deportes colectivos.

Por otro lado, respecto al espacio de juego, si existen multitud de investigaciones que analicen este aspecto (Castellano, Puente, Echeazarra, Usabiaga, y Casamichana, 2016; HillHaas, Coutts, Dawson, y Rowsell, 2010; Vilar, Duarte, Silva, Chow, y Davids, 2014). Todos ellos coindicen en que una mayor área de juego por jugador provoca mayores exigencias en frecuencia cardíaca, distancia total y distancia a alta intensidad. Por el contrario, un espacio más reducido de juego, provoca un mayor número de cambios de velocidad (aceleraciones y desaceleraciones). Además, cuando se comparan juegos reducidos con respecto a competición oficial se encontraron diferencias significativas en aceleraciones y desaceleraciones, siendo mayores estás exigencias en las tareas basadas en formas jugadas (Gómez-Carmona, Gamonales, Pino-Ortega et al., 2018). Por tanto, el uso de espacios reducidos debe ser utilizado con cautela, pudiendo provocar sobreentrenamiento en cambios de velocidad (Casamichana y Castellano, 2015), y por consiguiente un aumento del riesgo lesional (HillHaas, Dawson, Impellizzeri, y Coutts, 2011).

\title{
Implicación cognitiva
}

En cuanto a la carga psicológica de la tarea, se encuentran diferencias significativas en todas las variables analizadas con un tamaño del efecto grande (distancia total, Player Load y frecuencia cardíaca), moderado (distancia a alta intensidad) y bajo (aceleraciones). Los mayores valores se encuentran en las tareas donde existe una relación con más de dos compañeros, alcanzando el máximo valor cuando la relación es con todo el equipo. Los menores valores se encuentran en las tareas donde la relación es individual o con un compañero (parejas). En esta línea, el uso de metodologías constructivistas respecto a metodologías tradicionales encuentran que los modelos comprensivos (aprendizaje basado en el juego) obtienen mayores exigencias y motivación durante la práctica respecto a los tradicionales (eficacia técnica) debido a que durante la tarea se encuentra mayor: (1) nivel de

Para citar este artículo utilice la siguiente referencia: Gómez-Carmona, C.D.; Gamonales, J.M.; Feu, S.; Ibáñez, S.J. (2019). Estudio de la carga interna y externa a través de diferentes instrumentos. Un estudio de casos en fútbol formativo. Sportis Sci J, 5 (3), 444-468. 
Artículo Original. Estudio de la carga interna y externa a través de diferentes instrumentos. Un estudio de casos en fútbol formativo. Vol. 5 , n. ${ }^{\circ}$; p. 444-468, septiembre 2019. A Coruña. España ISSN 2386-8333.

oposición, (2) mayor tiempo de actividad (densidad y ejecución simultánea), (3) espacio de juego y (4) carga competitiva (González Espinosa et al., 2017).
\end{abstract}

\title{
Análisis relacional
}

Finalmente, los resultados muestran una fuerte relación entre las variables de carga objetiva (distancia, distancia a alta intensidad, aceleraciones y Player Load) respecto a la carga externa subjetiva a través de la categorización de las tareas $(r>0.84)$. En deportes de invasión, el indicador de carga externa subjetiva más utilizado ha sido la percepción subjetiva del esfuerzo (RPE) (Abbott et al., 2018; Casamichana et al., 2013), la cual ha reportado altas correlaciones tanto en entrenamiento como en competición $(r>.70)$ con diferentes variables de carga como frecuencia cardíaca, distancia recorrida o Player Load, entre otras. Aunque esta herramienta sea gratuita y fácil de registrar, existe la limitación de que no analiza las características de las tareas, sino que únicamente describe su carga física. Por tanto, la alta correlación obtenida por la herramienta SIATE, la sitúa como una alternativa más completa para el registro tanto de las demandas de carga como de las características pedagógicas y organizativas de las tareas en las sesiones de entrenamiento.

\section{Conclusiones}

La modificación de las variables de carga externa subjetiva provoca un efecto directo en las variables de carga externa (cinemáticas y neuromusculares) e interna. Por tanto, el diseño de las tareas de entrenamiento es un proceso muy importante para la planificación tanto de las demandas físicas como de los requerimientos pedagógicos debido a la influencia analizada tanto en el presente estudio como en investigaciones previas. Para su cuantificación y valoración, herramientas objetivas para su análisis como el Sistema Integral para el Análisis de las Tareas de Entrenamiento permiten registrar ambas variables (pedagógicas y de carga externa) de una forma útil y gratuita en el fútbol formativo, donde los recursos económicos son escasos para disponer de herramientas tecnológicas, debido a la alta relación existente entre ambos métodos.

Para citar este artículo utilice la siguiente referencia: Gómez-Carmona, C.D.; Gamonales, J.M.; Feu, S.; Ibáñez, S.J. (2019). Estudio de la carga interna y externa a través de diferentes instrumentos. Un estudio de casos en fútbol formativo. Sportis Sci J, 5 (3), 444-468. 
Artículo Original. Estudio de la carga interna y externa a través de diferentes instrumentos. Un estudio de casos en fútbol formativo. Vol. 5 , n. ${ }^{\circ}$; p. 444-468, septiembre 2019. A Coruña. España ISSN 2386-8333.
\end{abstract}

\title{
Referencias bibliográficas
}

Abbott, W., Brickley, G., y Smeeton, N. J. (2018). Positional Differences in GPS Outputs and Perceived Exertion During Soccer Training Games and Competition: Journal of

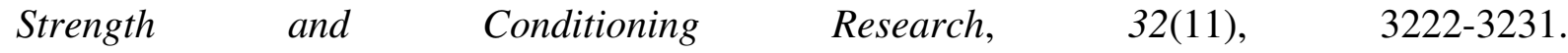
https://doi.org/10.1519/JSC.0000000000002387

Achten, J., y Jeukendrup, A. E. (2003). Heart rate monitoring. Sports Medicine, 33(7), $517-538$.

Akenhead, R., y Nassis, G. P. (2016). Training Load and Player Monitoring in HighLevel Football: Current Practice and Perceptions. International Journal of Sports Physiology and Performance, 11(5), 587-593. https://doi.org/10.1123/ijspp.2015-0331

Arruda, A. F. S., Carling, C., Zanetti, V., Aoki, M. S., Coutts, A. J., y Moreira, A. (2015). Effects of a Very Congested Match Schedule on Body-Load Impacts, Accelerations, and Running Measures in Youth Soccer Players. International Journal of Sports Physiology and Performance, 10(2), 248-252. https://doi.org/10.1123/ijspp.2014-0148

Ato, M., López-García, J. J., y Benavente, A. (2013). Un sistema de clasificación de los diseños de investigación en psicología. Anales de Psicología, 29(3), 1038-1059. https://doi.org/10.6018/analesps.29.3.178511

Bastida-Castillo, A., Gómez-Carmona, C. D., De la Cruz Sánchez, E., y Pino-Ortega, J. (2018). Accuracy, intra- and inter-unit reliability, and comparison between GPS and UWBbased position-tracking systems used for time-motion analyses in soccer. European Journal of Sport Science, 18(4), 450-457. https://doi.org/10.1080/17461391.2018.1427796

Bastida-Castillo, A., Gómez-Carmona, C. D., Hernandez, A., y Pino-Ortega, J. (2018). Validez y fiabilidad de un dispositivo inercial (WIMU PRO $^{\mathrm{TM}}$ ) para el análisis del posicionamiento en balonmano. E-Balonmano. com: Revista de Ciencias del Deporte, 14(1), 9-16.

Para citar este artículo utilice la siguiente referencia: Gómez-Carmona, C.D.; Gamonales, J.M.; Feu, S.; Ibáñez, S.J. (2019). Estudio de la carga interna y externa a través de diferentes instrumentos. Un estudio de casos en fútbol formativo. Sportis Sci J, 5 (3), 444-468. 
Artículo Original. Estudio de la carga interna y externa a través de diferentes instrumentos. Un estudio de casos en fútbol formativo. Vol. 5 , n. ${ }^{\circ}$; p. 444-468, septiembre 2019. A Coruña. España ISSN 2386-8333.

Bastida-Castillo, Alejandro, Gómez-Carmona, C. D., de la Cruz Sánchez, E., y PinoOrtega, J. (2019). Comparing Accuracy between Global Positioning Systems and UltraWideband-Based Position Tracking Systems Used for Tactical Analyses in Soccer. European Journal of Sport Science, Pendiente de Publicación.

Bradley, P. S., Sheldon, W., Wooster, B., Olsen, P., Boanas, P., y Krustrup, P. (2009). High-intensity running in English FA Premier League soccer matches. Journal of Sports Sciences, 27(2), 159-168. https://doi.org/10.1080/02640410802512775

Buchheit, M., Lacome, M., Cholley, Y., y Simpson, B. M. (2018). Neuromuscular Responses to Conditioned Soccer Sessions Assessed via GPS-Embedded Accelerometers: Insights Into Tactical Periodization. International Journal of Sports Physiology and Performance, 13(5), 577-583. https://doi.org/10.1123/ijspp.2017-0045

Cañadas, M., Rodríguez, G., Feu, S., Parejo, I., y García, J. (2013). Relathionship between pedagogical content knowledge and coaching methods. Revista de Psicología del Deporte, 22(1), 183-186.

Casamichana, D., y Castellano, J. (2015). The Relationship Between Intensity Indicators in Small-Sided Soccer Games. Journal of Human Kinetics, 46(1), 119-128. https://doi.org/10.1515/hukin-2015-0040

Casamichana, D., Castellano, J., Calleja-Gonzalez, J., San Román, J., y Castagna, C. (2013). Relationship Between Indicators of Training Load in Soccer Players. Journal of $\begin{array}{llll}\text { Strength } \quad \text { Conditioning 27(2), } & \text { 369-374. }\end{array}$ https://doi.org/10.1519/JSC.0b013e3182548af1

Castellano, J., Puente, A., Echeazarra, I., Usabiaga, O., y Casamichana, D. (2016). Number of Players and Relative Pitch Area per Player: Comparing Their Influence on Heart Rate and Physical Demands in Under-12 and Under-13 Football Players. PLoS ONE, 11(1), e0127505. https://doi.org/10.1371/journal.pone.0127505

Cohen, J. (1988). Statistical power analysis for the behavioral sciences (2nd ed.). Hillsdale, NJ: Erlbaum.

Para citar este artículo utilice la siguiente referencia: Gómez-Carmona, C.D.; Gamonales, J.M.; Feu, S.; Ibáñez, S.J. (2019). Estudio de la carga interna y externa a través de diferentes instrumentos. Un estudio de casos en fútbol formativo. Sportis Sci J, 5 (3), 444-468. DOI: https://doi.org/10.17979/sportis.2019.5.3.5464 
Artículo Original. Estudio de la carga interna y externa a través de diferentes instrumentos. Un estudio de casos en fútbol formativo. Vol. 5 , n. ${ }^{\circ}$; p. 444-468, septiembre 2019. A Coruña. España ISSN 2386-8333.

Cummins, C., Orr, R., O’Connor, H., y West, C. (2013). Global Positioning Systems (GPS) and Microtechnology Sensors in Team Sports: A Systematic Review. Sports Medicine, 43(10), 1025-1042. https://doi.org/10.1007/s40279-013-0069-2

Dellaserra, C. L., Gao, Y., y Ransdell, L. (2014). Use of Integrated Technology in Team Sports: A Review of Opportunities, Challenges, and Future Directions For Athletes. Journal of Strength and Conditioning Research, 2(28), 556-573.

Field, A. (2013). Discovering Statistics Using IBM SPSS Statistics (4. ${ }^{a}$ ed.). Londres: SAGE.

Gamonales, J. M., Gómez-Carmona, C. D., Córdoba-Caro, L. G., y Ibáñez, S. J. (2019). Influencia del perfil de entrenador en el diseño de las tareas en el fútbol. Estudio de caso. Journal of Sport and Health Research, 11(Supl 1), 69-82.

Gamonales, J. M., Gómez-Carmona, C. D., León, K., Muñoz-Jiménez, J., y Ibáñez, S. J. (2020). Estudio de las variables pedagógicas en tareas de entrenamiento en fútbol-base según el mesociclo competitivo. Un estudio de casos. Retos. Nuevas tendencias en Educación Física, Deporte y Recreación, Pendiente de Publicación.

Gamonales, J. M., Gómez-Carmona, C. D., León-Guzmán, K., García-Santos, D., Gamero-Portillo, M. D. G., y Muñoz-Jiménez, J. (2019). Análisis de las tareas de entrenamiento en fútbol-base: Diferencias entre dos meses durante el periodo competitivo en la categoría sub-19. Sportis. Scientific Journal of School Sport, Physical Education and Psychomotricity, 5(1), 30-52. https://doi.org/10.17979/sportis.2019.5.1.3469

Gamonales, J. M., Muñoz-Jiménez, J., León, K., y Ibáñez, S. J. (2017). Entrenamiento y confiabilidad entre observadores en el análisis del fútbol para ciegos (Reliability and intercoders training in the analysis of football for blind persons). Retos, (34), 155-161.

Gomez-Carmona, C. D., Bastida-Castillo, A., González-Custodio, A., Olcina, G., y Pino-Ortega, J. (2019). Using an inertial device (WIMU $\mathrm{PRO}^{\mathrm{TM}}$ ) to quantify neuromuscular load in running: Reliability, convergent validity and the influence of type of surface and

Para citar este artículo utilice la siguiente referencia: Gómez-Carmona, C.D.; Gamonales, J.M.; Feu, S.; Ibáñez, S.J. (2019). Estudio de la carga interna y externa a través de diferentes instrumentos. Un estudio de casos en fútbol formativo. Sportis Sci J, 5 (3), 444-468. DOI: https://doi.org/10.17979/sportis.2019.5.3.5464 
Artículo Original. Estudio de la carga interna y externa a través de diferentes instrumentos. Un estudio de casos en fútbol formativo. Vol. 5 , n. ${ }^{\circ}$; p. 444-468, septiembre 2019. A Coruña. España ISSN 2386-8333.

device location. The Journal of Strength and Conditioning Research, Pendiente de Publicación. https://doi.org/10.1519/JSC.0000000000003106

Gómez-Carmona, C. D., Gamonales, J. M., Pino-Ortega, J., y Ibáñez, S. J. (2018). Comparative Analysis of Load Profile between Small-Sided Games and Official Matches in Youth Soccer Players. Sports, 6(4), 173. https://doi.org/10.3390/sports6040173

Gómez-Carmona, C. D., Bastida-Castillo, A., García-Rubio, J., Ibáñez, S. J., y PinoOrtega, J. (2019). Static and dynamic reliability of WIMU PROTM accelerometers according to anatomical placement. Proceedings of the Institution of Mechanical Engineers, Part P: Journal of Sports Engineering and Technology, 233(2), 238-248. https://doi.org/10.1177/1754337118816922

Gómez-Carmona, C. D., García-Rubio, J., Muñoz-Jiménez, J., y Gamonales, J. M. (2018). Relación entre el medio de iniciación al entrenamiento y las variables pedagógicas que definen las tareas en el fútbol. TRANCES. Transmisión del Conocimiento Educativo y de la Salud, 10(Suppl.1), 401-420.

González-Espinosa, S., Ibáñez, S. J., Feu, S., y Galatti, L. R. (2017). Programas de intervención para la enseñanza deportiva en el contexto escolar, PETB y PEAB: Estudio preliminar. Retos. Nuevas tendencias en Educación Física, Deporte y Recreación, (31), 107113.

Gracia, F., Garcia-Rubio, J., Cañadas, M., y Ibáñez, S. J. (2014). Heart rate differences in small sided games in formative basketball. E-balonmano. com: Revista de Ciencias del Deporte, 10(1), 23-30.

Halouani, J, Chtourou, H., Dellal, A., Chaouachi, A., y Chamari, K. (2017). Soccer small-sided games in young players: rule modification to induce higher physiological responses. Biology of Sport, 34(2), 163-168. https://doi.org/10.5114/biolsport.2017.64590

Halouani, Jamel, Chtourou, H., Gabbett, T., Chaouachi, A., y Chamari, K. (2014). Small-Sided Games in Team Sports Training: A Brief Review. Journal of Strength and Conditioning Research, 28(12), 3594-3618. https://doi.org/10.1519/JSC.0000000000000564

Para citar este artículo utilice la siguiente referencia: Gómez-Carmona, C.D.; Gamonales, J.M.; Feu, S.; Ibáñez, S.J. (2019). Estudio de la carga interna y externa a través de diferentes instrumentos. Un estudio de casos en fútbol formativo. Sportis Sci J, 5 (3), 444-468. 


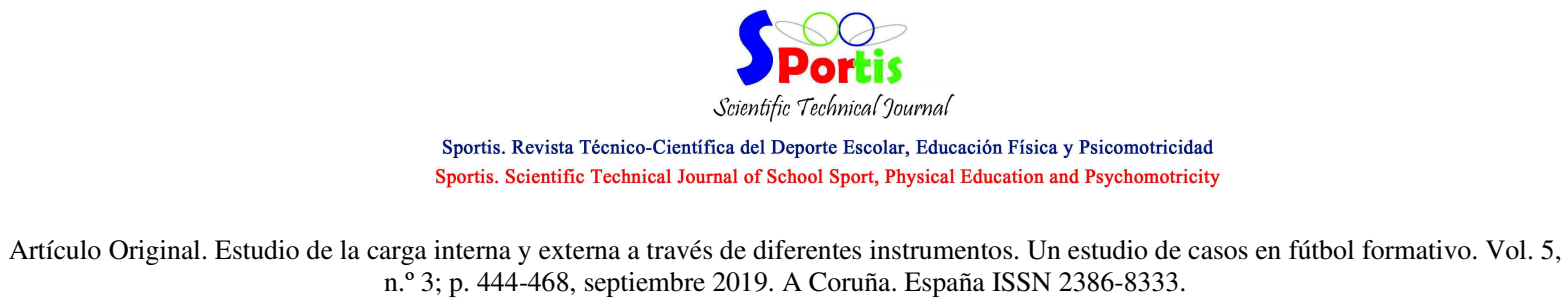

Hill-Haas, S. V., Coutts, A. J., Rowsell, G. J., y Dawson, B. T. (2009). Generic Versus Small-sided Game Training in Soccer. International Journal of Sports Medicine, 30(9), 636642. https://doi.org/10.1055/s-0029-1220730

Hill-Haas, Stephen V., Coutts, A. J., Dawson, B. T., y Rowsell, G. J. (2010). Timemotion characteristics and physiological responses of small-sided games in elite youth players: The influence of player number and rule changes. Journal of Strength and Conditioning Research, 24(8), 2149-2156. https://doi.org/10.1519/JSC.0b013e3181af5265

Hill-Haas, Stephen V., Dawson, B., Impellizzeri, F. M., y Coutts, A. J. (2011). Physiology of small-sided games training in football. Sports medicine, 41(3), 199-220. https://doi.org/10.2165/11539740-000000000-00000

Ibáñez, S. J., Feu, S., y Cañadas, M. (2016). Sistema Integral para el Análisis de las Tareas de Entrenamiento, SIATE, en Deportes de Invasión [Integral analysis system of training tasks, SIATE, in invasion games]. E-Balonmano.com: Revista de Ciencias del Deporte, 12(1), 3-30.

Köklü, Y., Ersöz, G., Alemdaroğlu, U., Aşç, A., y Özkan, A. (2012). Physiological Responses and Time-Motion Characteristics of 4-A-Side Small-Sided Game in Young Soccer Players: The Influence of Different Team Formation Methods. Journal of Strength and Conditioning Research, 26(11), 3118-3123. https://doi.org/10.1519/JSC.0b013e318244341d

Molina-Carmona, I., Gomez-Carmona, C. D., Bastida-Castillo, A., y Pino-Ortega, J. (2018). Validez del dispositivo inercial WIMU PRO ${ }^{\mathrm{TM}}$ para el registro de la frecuencia cardíaca en un test de campo. Sport-TK: Revista Euroamericana de Ciencias del Deporte, 7 , 81-86.

Pino-Ortega, J., Rojas-Valverde, D., Gómez-Carmona, C. D., Bastida-Castillo, A., Hernández-Belmonte, A., García-Rubio, J., ... Ibáñez, S. J. (2019). Impact of Contextual Factors on External Load During a Congested-Fixture Tournament in Elite U'18 Basketball Players. Frontiers in Psychology, 10, 1100. https://doi.org/10.3389/fpsyg.2019.01100

\footnotetext{
Para citar este artículo utilice la siguiente referencia: Gómez-Carmona, C.D.; Gamonales, J.M.; Feu, S.; Ibáñez, S.J. (2019). Estudio de la carga interna y externa a través de diferentes instrumentos. Un estudio de casos en fútbol formativo. Sportis Sci J, 5 (3), 444-468. DOI: https://doi.org/10.17979/sportis.2019.5.3.5464 
Artículo Original. Estudio de la carga interna y externa a través de diferentes instrumentos. Un estudio de casos en fútbol formativo. Vol. 5 , n. ${ }^{\circ}$; p. 444-468, septiembre 2019. A Coruña. España ISSN 2386-8333.

Reche-Soto, P., Cardona-Nieto, D., Diaz-Suarez, A., Bastida-Castillo, A., GomezCarmona, C., Garcia-Rubio, J., y Pino-Ortega, J. (2018). Player Load and Metabolic Power Dynamics as Load Quantifiers in Soccer. Journal of Human Kinetics, Pendiente de Publicación. http://www.doi.org/10.2478/hukin-2018-0072

Reche-Soto, P., Cardona-Nieto, D., Diaz-Suarez, A., Gomez-Carmona, C., y PinoOrtega, J. (2019). Demandas tácticas de juegos reducidos en fútbol: influencia de la tecnología utilizada. Revista Internacional de Medicina y Ciencias de la Actividad Física y del Deporte, Pendiente de publicación.

Torres-Ronda, L., Gonçalves, B., Marcelino, R., Torrents, C., Vicente, E., y Sampaio, J. (2015). Heart Rate, Time-Motion, and Body Impacts When Changing the Number of Teammates and Opponents in Soccer Small-Sided Games: Journal of Strength and Conditioning Research, 29(10), 2723-2730. https://doi.org/10.1519/JSC.0000000000000932

Vilar, L., Duarte, R., Silva, P., Chow, J. Y., y Davids, K. (2014). The influence of pitch dimensions on performance during small-sided and conditioned soccer games. Journal of Sports Sciences, 32(19), 1751-1759. https://doi.org/10.1080/02640414.2014.918640

White, A., Hills, S. P., Cooke, C. B., Batten, T., Kilduff, L. P., Cook, C. J., ... Russell, M. (2018). Match-Play and Performance Test Responses of Soccer Goalkeepers: A Review of Current Literature. Sports Medicine, 48(11), 2497-2516. https://doi.org/10.1007/s40279-0180977-2

Para citar este artículo utilice la siguiente referencia: Gómez-Carmona, C.D.; Gamonales, J.M.; Feu, S.; Ibáñez, S.J. (2019). Estudio de la carga interna y externa a través de diferentes instrumentos. Un estudio de casos en fútbol formativo. Sportis Sci J, 5 (3), 444-468. 